\title{
Einführung: Migration als Herausforderung für Europa
}

Magdalena Nowicka

Fachgebiet: Migration

\section{Erstveröffentlicht in:}

Nowicka, Magdalena (2007): Einführung: Migration als Herausforderung für Europa. In: Nowicka, Magdalena (Hrsg.): Von Polen nach Deutschland und zurück. Die Arbeitsmigration und ihre Herausforderungen für Europa, Bielefeld: transcript Verlag, S. 7-22

ISBN 978-3-89942-605-2

The final publication is available at transcript via http://www.transcriptverlag.de/978-3-89942-605-2/von-polen-nach-deutschland-und-zurueck\#

(C) 2007 transcript Verlag, Bielefeld

This document is the author's final version of the published version. There may be differences between this version and the published version. You are advised to consult the publisher's version if you wish to cite from it.

\section{Prof. Dr. Magdalena Nowicka}

Professur für Migration und Transnationalismus

Humboldt-Universität zu Berlin

Institut für Sozialwissenschaften

Tel: 0049 (30) 20934239

Fax: 0049 (30) 20934519

magdalena.nowicka@hu-berlin.de

\section{Arbeits- und Forschungsschwerpunkte}

Soziologische Migrationsforschung, soziale Ungleichheit in transnationaler Perspektive, Mobilitätsparadigma, Kosmopolitismus, Theorien der Modernisierung und Globalisierung, Transnationalismus, qualitative Methoden, empirische Forschung 


\section{EINFÜHRUNG: \\ MIGRATION ALS HERAUSFORDERUNG FÜR EUROPA MAGDALENA NOWICKA}

\section{Die Invasion und die Flut}

Am 1. Mai 2007 ist der dritte Jahrestag der so genannten EU-Osterweiterung. 2004 wurden 10 neue Länder in die Europäische Union aufgenommen: Estland, Lettland, Litauen, Malta, Polen, die Slowakei, Slowenien, die Tschechische Republik, Ungarn und Zypern. Dabei ist Polen mit knapp über 38 Millionen Einwohnern das größte Beitrittsland. Sein Beitritt in die EU hat widersprüchliche Reaktionen hervorgerufen.

Nach drei Jahren scheint aber die öffentliche Diskussion über die Folgen des Beitritts etwas gedämpft zu sein, vor allem im Vergleich zu den ersten Meldungen und der großen Aufregung über die „polnische Welle“. Der erste Jahrestag wurde in Deutschland zu einer Gelegenheit, noch einmal über die Ängste von Lohndumping und der Flut von polnischen Handwerkern zu schreiben. So berichtete Ende April 2005 der Stern über die Konkurrenz aus dem Osten - polnische Alleskönner, die den Deutschen die Arbeit auf Baustellen oder bei Renovierungen in privaten Haushalten wegnehmen. Und das alles völlig legal (!). Die polnischen Fliesenleger wurden zum Sinnbild für die Gefahr aus dem Osten, die die Erweiterung der EU gnadenlos ausnutzen (Berliner Zeitung 04.05.2005; Stern 17/2005). Die Situation wurde in den Zeitungen als eine unerwartete Entwicklung, eine Überraschung dargestellt, etwas, was man kaum vorhersehen könnte: die Zeit schrieb „so hat sich Wolfgang Clement das vermutlich nicht vorgestellt", als er zu mehr Selbständigkeit in Deutschland aufrief (Die Zeit 12/2005). Die Empörung über die neue Situation spiegelte die Tatsache wider, dass vor der Erweiterung der EU kaum eine Diskussion über die Europäische (soziale) Integration stattgefunden hatte. Damals fokussierte man sich ausschließlich auf die „Schutzmaßnahmen“ - die Übergangsregelungen, die den einheimischen Arbeitsmarkt von den polnischen Arbeitern abschirmen sollten. Es scheint, dass die Einführung der Niederlassungsfreiheit für viele tatsächlich eine Überraschung war und deswegen als eine „Lücke im EU-Beitrittsgesetz“ (Berliner Zeitung 04.05.2005) beschrieben wurde. Als Reaktion darauf vermehrten sich die Vermutungen, dass die meisten Polen sowieso illegal, da als Scheinselbständige, arbeiten.

Die Angst um die Arbeitsplätze der Deutschen (Stern 13/2005) vermischte sich mit der Besorgnis über die Bedingungen, in denen die Billigarbeiter in Deutschland leben. Sie werden nur in bar bezahlt, verdienen sehr wenig, sind den unehrlichen Arbeitgebern völlig ausgesetzt, haben keine soziale Absicherung und wohnen in dramatisch schlechten Verhältnissen, auf 
Feldbetten in überfüllten Wohnungen oder Kellern (FAZ 17.05.2005). Für die für alle Betroffenen - die deutschen und die polnischen Arbeiter - schwierige und unangenehme Situation wurden alle mögliche Instanzen beschuldigt, von der Globalisierung und der Osterweiterung über die deutsche Regierung, die keine entsprechende Schritte dagegen unternahm und sogar die Handwerksordnung gelockert hat bis zu den gewinnsüchtigen Besitzern der Fleischbetriebe und den polnischen Arbeitern selbst.

Dagegen vermehrten sich 2006 die Zeitungsartikel in Polen, die über die Diskriminierung von polnischen Arbeitnehmern und Unternehmen auf den Arbeitsmärkten der alten EU berichteten. Man sprach von „Überfällen“ auf deutsche Baustellen, von Zollbeamten mit Hunden wie in einem Kriegsfilm, Durchsuchungen, Unterstellungen, von in den Ruin getriebenen Unternehmen und dem rücksichtlosen Kampf um Aufträge (Gazeta Wyborcza 27.06.2006).

Tatsächlich wandelte sich die primär auf die Erweiterung und die Integration (der Märkte) der EU gerichtete Diskussion im Laufe des Jahres 2006 zur Diskussion über die zunehmend schwierige Beziehung zwischen Polen und Deutschland. Nicht ohne Grund wurde 2006 zum DeutschPolnischen Jahr ernannt und von mehreren Veranstaltungen (vor allem Kunstausstellungen) in beiden Ländern begleitet. Fast drei Jahre nach der Osterweiterung schreiben jedoch die Zeitungen der benachbarten Staaten weiter über Karikaturen und Satiren, abgesagten Treffen und gegenseitigen Missverständnissen. Nicht nur hat sich die Sprache verschärft, auch die Art, wie die Probleme behandelt werden. Neulich haben drei Familien polnischer Herkunft eine Klage beim Europäischen Parlament eingereicht, in der sie Deutschland der Germanisierung ihrer Kinder beschuldigen (Gazeta Wyborcza 31.01.2007).

Von einer sachlichen und seriösen Betrachtung einer gemeinsamen Zukunft und des Zusammenlebens von Polen und Deutschen in Europa sind diese Meldungen weit entfernt, geschweige denn von einem Dialog. Die deutsche Politik hat praktisch automatisch über die Verlängerung der Übergangsfristen für die nächsten zwei Jahre (bis zum 30.04.2009) entschieden. Auch die Wissenschaft hält sich hier zurück. Drei Jahre scheinen zu kurz zu sein, um die Folgen für die deutsche Wirtschaft und Gesellschaft zu beurteilen, vor allem weil nur das erste Jahr nach der Osterweiterung bis jetzt statistisch vollständig erfasst wurde. Eine Überprüfung der wissenschaftlichen Vorhersagen bezüglich des Volumens der Migration aus Mittel- und Osteuropa würde aus diesem Grund schwierig, wobei die bisher verfügbaren Daten suggerieren, dass sich die meisten Schätzungen geirrt haben. Nicht zuletzt muss man anmerken, dass die wissenschaftlichen Analysen auch gewissermaßen konjunkturell sind und dem öffentlichen Interesse folgen. So steht die türkische und nicht die polnische Bevölkerung in Deutschland im Fokus. 
Ähnlich geben die innenpolitischen Kämpfe der öffentlichen Diskussionen in Polen die Richtung. So werden die Argumente gegen und für den polnischen Präsidenten Kaczyński und seine Außenpolitik mit Berichten über die Situation der Polen und der polnischen Unternehmen in Deutschland vermischt. Die Medien werden aber von Artikeln und Reportagen über die neue Welle der Migranten nach Großbritannien und Irland dominiert. Sowohl dieses Thema als auch die neue Zuwanderung aus dem Osten, vor allem aus der Ukraine, werden als gesellschaftlich wichtig und interessant betrachtet. Diesem Interesse folgt ebenfalls die Migrationsforschung.

Die öffentliche Diskussion der letzten drei Jahre sollte man nicht als eine wissenschaftlich irrelevante Sammlung von Vorurteilen und Panikmache verstehen. Die Zeitungsartikel spiegeln ein interessantes und wichtiges Phänomen wider. Traditionell war die Vorstellung des Zusammenlebens in Europa ein Ausdruck der nationalen Werte und Imperative. Innerhalb des nationalen „Behälter"-Modells der Kultur wurden die öffentlichen Diskurse um die nationale Mehrheitsgesellschaft und ihre Minderheiten aufgebaut - die Minderheiten, die als Migranten, Fremde oder Ausländer konzipiert wurden (Hahn 1994; Radtke 1996; Bielefeld 1998; Bauer et al. 2002). Diese Diskurse sind unverändert dominant, auch in dem Kontext, wenn Migranten, zum Beispiel Polen, von innerhalb der Europäischen Union kommen und eigentlich als EU-Bürger den Deutschen, den Briten oder den Franzosen gleich sind. Sie unterscheiden zwischen „uns Deutschen“ und „den Polen“ (,uns Polen“- ,den Deutschen") und isolieren anhand von statistischen Daten und mehr oder wenigen objektiven Erzählungen und Fakten eine bestimmte Gruppe (Migranten) innerhalb der Mehrheitsgesellschaft.

Analysiert man diese Diskurse aus der Perspektive der Aufnahmegesellschaft, so wird sichtbar, dass sie als ein Schutzmechanismus der existenziellen Sicherheit der Mehrheit der nationalen Bevölkerung und des Umgangs mit Bedrohungen dieser Sicherheit funktionieren. Von den Wissenschaftlern (auch oder sogar primär in Polen) hörte man die „banale Wahrheit“: Die Folgen der EU-Osterweiterung sind nicht gleich verteilt. Auf der Makro-Skala bringt die Erweiterung für alle - die „alten“ wie die „neuen“ Länder - Vorteile; bestimmte Gruppen werden sich aber „anpassen“ müssen, sie werden die Erweiterung als Nachteil empfinden. Die Meldungen über Lohndumping, verlorene Arbeitsplätze, Kriminalität der Migranten oder die Bedrohung für die Sozialsysteme sind aber nicht nur ein Zeichen dafür, dass sich tatsächlich manche Gruppen als Verlierer fühlten. Sie zeigen das Gefühl des Verlusts der Kontrolle über das Geschehen im eigenen Land und in Europa. Die Postulate der verschärften Kontrolle durch Zoll und Polizei, die Appelle an die Politiker für die Einführung eines Mindestlohns und die Verlängerung der Übergangsfristen sind ein Ausdruck dieses Wunsches und der Versuch, die Kontrolle zurück zu gewinnen. Diese ist in einem tatsächlich offenen 
europäischen Raum zunehmend schwierig, zumindest wenn man sie mit ,alten“ Mechanismen herstellen wird. Diese nationale Logik verhindert die Vorstellung eines breiten europäischen, inklusiven Raums, in dem die Individuen tatsächlich frei wählen, wo sie arbeiten und wo sie wohnen wollen. Die Verschiebung der Diskussion auf die bilateralen Beziehungen zwischen Polen und Deutschland ist ein Zeichen dafür, dass es an möglichen Mustern und Beschreibungen mangelt, mit deren Hilfe man in der Öffentlichkeit das Zusammenleben in Europa entsprechend reflektieren könnte.

Die Zeitungsmeldungen aus den letzten Jahren, unabsichtlich und der nationalen Logik entsprechend, verkennen die Tatsache, dass die polnische Migration keine neue, erst nach der Osterweiterung entstandene Erscheinung ist, sondern ein stabiles und sehr ausdifferenziertes Phänomen. Andererseits aber weisen sie auf etwas Wichtiges hin, auf eine tatsächlich neue Entwicklung. Sie berichten über Menschen, die Initiative ergreifen, eine Stadt verlassen und woanders einen Job finden, ohne dabei auf die Freundschaften, die eigene Sprache oder Kultur zu verzichten. Sie zeigen, dass es für sehr viele, vor allem junge Polen, selbstverständlich ist, ihren Lebensraum über die nationalen Grenzen hinweg zu erweitern. Sie nutzen die Chancen, die ihnen die Osterweiterung der EU gibt, um der Arbeitslosigkeit oder niedrigen Lohnen und mangelnden Berufschancen zu entkommen. Sie gewinnen die Kontrolle über ihr eigenes Leben dadurch zurück, dass sie sich in mehr als einem Land verorten. Für diese „existenziellen Unternehmen“ ist Migration und Transnationalität in Europa eine Schlüsselressource.

\section{Migration und Mobilität in Europa}

Europa ist sowohl ein gemeinsames Projekt seiner Mitgliedstaaten als auch ein Lebensraum für ihre Bevölkerungen. Die Europäisierung - der Prozess des Zusammenwachsens nationaler Gesellschaften zu einem qualitativ neuen Gebilde - wird einerseits von den Institutionen der EU vorangetrieben und andererseits entsteht sie von „unten“, durch die Verbindungen zwischen ihren Einwohnern. Dabei wird der Mobilität von Personen eine besondere Rolle zugeschrieben. Die räumliche Bewegung der Menschen wird einerseits als ein Grund und Antrieb der Integration in Europa und andererseits als ihre Folge gesehen (Eder 2000; Jordan/Düvell 2002; Beck/Grande 2004; Verwiebe 2004). Diese Sicht teilen sich Wissenschaft und Politik. Das Grundprinzip der EU ist, einen einheitlichen gemeinsamen Markt zu bilden, auf dem sich Kapital, Waren und Arbeitskraft bewegen können. Dieses Ziel bediente die Neudefinition der Migration zwischen den Mitgliedsstaaten der EU als Mobilität (Heckmann/Tomei 1996). Mobilität wird gleichzeitig mit Entscheidungsfreiheit verbunden, als etwas, was den Individuen eine schnelle 
Anpassung an neue ökonomische Bedingungen ermöglicht. Migration wird zu einem Begriff, mit dem man Zuwanderung von außerhalb der EU beschreibt. Diese Bewegungen sollen reguliert und kontrolliert werden, um zum Beispiel den saisonalen Arbeitskraftbedarf zu decken. So wird Migration als „Notfalllösung“ betrachtet, falls die Binnenmobilität in der EU nicht ausreicht.

Trotz aller Bemühungen (wie dem Schengen-Abkommen) bleibt die innereuropäische Mobilität gering und geht sogar zurück (Jordan/Düvell 2002: 36). Andererseits befürchtet man seit den 1990er die möglichen negativen Folgen der Mobilität, sowohl in Bezug auf das Wachsen der Gefälle der wirtschaftlich schwachen Regionen (Straubhaar 2000) als auch auf die Verschlechterung der Lebenschancen weniger mobiler Personen - Mobilität ist gesellschaftlich spaltend, bemerkt die European Foundation (1990: 10). Ferner ist man um das europäische Sozialmodell besorgt, so dass in den letzten Jahren neue Strategien entwickelt wurden, um die soziale Kohäsion und Inklusion zu gewährleisten (Jordan/Düvell 2002: 38). Dadurch sollten vor allem die negativen Folgen temporärer Wanderung der Arbeitskräfte minimiert werden. Je strenger die Zuwanderungspolitik und die Kontrollen, desto häufiger kommt es paradoxerweise $\mathrm{zu}$ unvollständiger, irregulärer Migration, die durch Globalisierung zu einem selbstverständlichen Teil des Alltags geworden ist.

Die geographische Bewegung von Menschen zwischen Staaten offenbart die Spannung zwischen den nationalen Prinzipien der Zuordnung zu einem Staat (Miera 1997) und der nationalstaatlichen Regulierung des Arbeitsmarkts, dem Prinzip der unbeschränkten Mobilität innerhalb der EU und den tatsächlichen Praktiken der Migranten. Hier ist das Beispiel der polnischen Arbeitskräfte sehr interessant - zwar ist Polen Mitglied der EU und ein Teil ihres Binnenmarkts, die Wanderungen der Arbeitskräfte werden jedoch als (kontrollbedürftige) Migration und nicht (erwünschte) Mobilität betrachtet. Für die Nationalstaaten stellt die geographische Mobilität ein Dilemma dar - sie ziehen einerseits die ausländischen Arbeitskräfte an, müssen ihnen aber andererseits gerade deswegen soziale Leistungen gewähren, das heißt gegen das eigene Prinzip der Geschlossenheit mit den Nicht-Bürgern solidarisch sein und sie gleich behandeln (Brauer/Faist/Jordan 1994). Sie müssen über die Inklusion und Exklusion bestimmter Gruppen entscheiden. Das Problem der Integration der Migranten bezieht sich auf diese Entscheidung. In vielen Ländern der EU wird die Frage der Integration unterschiedlich behandelt. Aber auch in einem Land haben Migranten verschiedener Herkunft, wie zum Beispiel Türken, Iraker oder Kenianer, in Deutschland unterschiedliche Stellung.

Sogar Migranten aus einem Land werden in Deutschland unterschiedlich gestellt, z.B. Studenten, Selbständige, Asylanten, Aussiedler oder Arbeitnehmer. Auf Grund des Prinzips der biologischen Abstammung (Bös 1993) wird den (Spät-)Aussiedlern eine Verbundheit und Loyalität zu 
Deutschland unterstellt und kulturelle und sozioökonomische Bindungen zum Herkunftsland dagegen tendenziell negiert (Miera 1997: 233). Der deutsche Staat fühlt sich ihnen verbunden - sie werden durch ihn gleich, anfänglich sogar privilegiert behandelt - ihnen werden nach der Einreise nach Deutschland Eingliederungshilfen angeboten, z.B. Sprachförderung oder Sozialhilfe (Bade/Oltmer 1999; Silbereisen et al. 1999; Strobl/Kühnel 2000). Seit 1993 gehören die Migranten aus Polen nicht mehr zu der Gruppe der Spätaussiedler. Die von ihnen, die das Kriterium der deutschen Staatsangehörigkeit nicht erfüllen, gehören zwar nicht zur Nation, können jedoch ihren Aufenthaltsstatus in Deutschland schrittweise im vorgesehenen Rahmen konsolidieren.

Verlässt man die nationalstaatliche Perspektive, so öffnen sich zwei Möglichkeiten - eine supranationale (der EU) und eine transnationale. Hier handelt sich um Bewegungen, die trotz oder gerade wegen der staatlichen Kontrollmechanismen und Politiken stattfinden. Diese Bewegungen können verschiedene Formen annehmen, von Aus- und Einwanderung mit der Absicht der permanenten Ansiedlung im Zielland zur unvollständigen, temporären, Pendel- oder Rotationsmigration (Sheffer 1989; Morokvasic/de Tinguy 1993; Cyrus 1994; Glick Schiller et al. 1995; Lie 1995). In Europa dominiert heutzutage die temporäre Migration bzw. Mobilität, wobei diese Art der Bewegungen nicht neu ist. Trotzdem ist sie erst seit den 1990er Jahren im Fokus, weil sie die Logik der nationalstaatlichen dualistischen Zuordnung der Zugehörigkeit zum Herkunfts- oder Zielland in Frage stellt. Damit werden auch die Grenzen aller Politiken sichtbar, die sich auf einer klaren Unterscheidung von den dazu- und den nicht-dazu-Gehörigen stützen - der Immigrations-, Arbeitsmarkt- und Sozialpolitik. Man kann sich einen polnischen Staatsbürger vorstellen, der in Polen Sozialleistungen bezieht, aber gleichzeitig illegal in Deutschland arbeitet oder jemanden, der zwar in Deutschland legal arbeitet, aber sein Geld in Polen regelmäßig ausgibt, wo er ein Haus besitzt, in dem er jedes Wochenende wohnt und sich dort mit seinen ausschließlich polnischen Freunden trifft und wo er auch nach der Pensionierung hinziehen möchte. Solche Situationen und Prozesse erforderten eine Reaktion - eine institutionelle Anpassung nationalstaatlichen Handelns. Das geschah bereits auf der Ebene der EU, z.B. in Form der Koordinierung der Sozialversicherungssysteme (VO (EWG) Nr. 1408/71). Eine Reaktion auf die Herausforderung der Migration sind auch die Versuche, die (nationalstaatliche) Kontrolle darüber zurück zu gewinnen, bzw. ihre negativen Folgen zu minimieren. Hier wird sowohl auf der Seite des Aufnahme- als auch des Herkunftslands verschiedenes unternommen. Die Polnische Nationalbank und das Zentrale Amt für Statistik sowie das Ministerium für Arbeit und Soziales geben offen zu, dass „die Realität rätselhaft geworden ist", weil man die Migrantenströme nicht erfassen kann. Es wurde von diesen Institutionen sogar eine Arbeitsgruppe ins Leben gerufen, um eine Methodologie zu entwickeln, mit deren Hilfe man die Geldtransfers der 
Migranten und ihren Einfluss auf Inflation und Konjunktur schätzen kann (Gazeta Wyborcza 26.04.2006; 27.06.2006). Andererseits versucht man die Migration zu steuern, zum Beispiel die Migranten zur Rückkehr durch günstige Immobilienkredite und neue Arbeitsplätze zu animieren (Gazeta Wyborcza 28.07.2006; 26.10.2006). Die Öffnung der Grenzen innerhalb der EU macht diese Situation noch schwieriger, vor allem, wenn viele Kompetenzen von den Nationalstaaten auf die Institutionen der EU übertragen werden.

Diese tatsächliche doppelte Verortung - in mehr als einem Land - wirft die Frage auf, was die Integration der Migranten in der Aufnahmegesellschaft bedeutet. Die Ängste um Desintegration der Gesellschaft und die Befürchtung, dass in Deutschland bereits Parallelgesellschaften entstanden sind, zeigen, dass diese Frage höchst aktuell ist und nicht nur die Wissenschaft, sondern auch die Öffentlichkeit beschäftigt. Gleichzeitig ist aber das Wissen über die transnationale Lebensführung und ihre Auswirkungen auf die Migranten und die Mehrheitsgesellschaften, in denen sie verortet sind, sehr gering.

\section{Themen und Perspektiven des Buchs}

Diese Fragen und Themen wurden nur zu selten in den Fokus gestellt. Mit diesem Band möchten wir sie aufgreifen und zu einer Diskussion über die Herausforderungen der Migration für die Zukunft der europäischen Gesellschaft animieren. Der Fokus auf Migration zwischen Polen und Deutschland ist aus mehreren Gründen gefallen - die geographische Nähe und eine lange bilaterale Geschichte führten dazu, dass sich zwischen Polen und Deutschland ein Migrationssystem entwickelte; die Osterweiterung war in Deutschland mit vielen Ängsten verbunden, die bis heute eine Rolle spielen; die politische Beziehung zwischen beiden Ländern hat sich von den gesellschaftlichen Zusammenhängen im letzten Jahr entkoppelt; nicht zuletzt schaffte der Beitritts Polens in die EU eine einmalige Situation, in der die Bewegungen zwischen den Ländern sowohl die Eigenschaften der Mobilität als auch der Migration haben.

Dabei möchten wir einen ausdifferenzierten Blick auf eine Gruppe richten, die oft vereinheitlich und vereinfacht dargestellt wird, aber selten in der Öffentlichkeit und der Politik präsent ist. Die polnische Migration nach Deutschland findet aus Sicht der Deutschen im Schatten der anderen Zuwanderungströme statt, vor allem der türkischen Einwanderung. Polen sind auch oft „unsichtbar“, weil sie den Deutschen ähnlich sehen, gut deutsch sprechen und ihre Kinder überdurchschnittlich gute Ergebnisse in der Schule erzielen (Lehmann et al. 2000). Die Polen in Deutschland bilden eine besonders vielfältige Gruppe - von den Enkeln der Zuwanderer am Ende des neunzehnten und am Anfangs des zwanzigsten Jahrhunderts, den Vertriebenen nach dem 
Zweiten Weltkrieg, den Spätaussiedlern bis zu den neuen Arbeitsmigranten der letzten 16 Jahre (Mrowka 2000). Heutzutage gibt es allerdings in Deutschland kaum Repräsentanten der politischen Auswanderung, die Menschen um sich gruppieren und organisieren und den polnischen Zuwanderern eine Stimme geben würden. Im Gegensatz zur Immigration in den USA oder der neuesten Immigration in Großbritannien sind Polen in Deutschland eine zersplitterte Gemeinde ohne klare Eliten, die weitgehend offen ist. Diese interne Ausdifferenzierung und die geographische Nähe zu Polen ist ein Grund dafür, dass Polen in Deutschland ihre Interessen nicht als eine Gruppe vorlegen und letztendlich tatsächlich „unsichtbarer“ erscheinen (Krasnodębski 2001).

Nimmt man die Gründe der Wanderungen als einen Anhaltspunkt, so untersuchen die Beiträge überwiegend die Arbeitsmigration. Dennoch möchten wir betonen, dass die polnische Migration verschiedene Formen annimmt, sowohl bezüglich der Dauer des Aufenthalts als auch Formen der Beschäftigung in Deutschland und der Bindung zu Polen.

In dem Band nehmen wir verschiedene Perspektiven in Anspruch: eine geographische, soziologische und ökonomische. Jede davon bringt andere Aspekte zum Vorschein, so dass wir ein erschöpfendes Bild der Wanderungen zwischen Polen und Deutschland zeigen können. Die in den Beiträgen präsentierten empirischen Untersuchungen fokussieren sich auf die Zeit nach der Osterweiterung der EU. Uns ist es jedoch wichtig, dass die heutige Migration ein Ergebnis langjähriger Prozesse ist, die sowohl in die Vergangenheit als in die Zukunft greifen. Viele der untersuchten Migranten hielten sich bereits vor dem Beitritt Polens in die EU in Deutschland auf; die Erweiterung der EU hat die Bedingungen der Transnationalisierung verändert und den Migranten neue Perspektiven geöffnet, die wir aufzeigen wollen.

\section{Arbeitsmigration in Kontexten}

Das Ziel des ersten Teils des Buchs ist, die Arbeitsmigration aus Polen in verschiedenen Kontexten zu präsentieren. Fokussiert man sich auf eine bestimmte Gruppe, so stellt sich die Frage, ob das untersuchte Phänomen eine Ausnahme oder ein typischer Trend ist und ob man Vergleiche mit anderen Gruppen ziehen kann. Im Hinblick auf die öffentlichen Diskussionen ist es interessant zu fragen, welche Stellung die polnische Arbeitsmigration in Deutschland, zum Beispiel im Vergleich zu den anderen Migrantengruppen, und in Europa, in Verbindung zur Wanderung in die Länder, die ihre Arbeitsmärkte öffneten, hat.

Barbara Dietz stellt in ihrem Beitrag die polnische Arbeitsmigration als Teil der Zuwanderung aus Mittel- und Osteuropa vor. Sie stellt eine der am stärksten wachsenden Ausländergruppen seit Beginn der neunziger Jahre dar. Dabei bespricht der Beitrag detailliert die Aufnahmebedingungen für mittel- 
und osteuropäische Zuwanderer, vor allem ihre rechtlichen Grundlagen vor und nach der EU-Osterweiterung. Hier wird die Zuwanderung aus Mittel- und Osteuropa als ein kontinuierlicher Trend gezeigt. Alle in dem Beitrag präsentierten Daten beziehen sich auf dem Zeitraum von 1989 bis einschließlich 2005. Barbara Dietz zeigt die demographische Charakteristik der Mittel- und Osteuropäer, die nach Deutschland kommen. Ihre Stellung auf dem Arbeitsmarkt in Deutschland wird sowohl mit den Deutschen als auch mit den Migrantengruppen aus den traditionellen Anwerbeländern verglichen.

Der Beitrag von Elmar Hönekopp beschäftigt sich ebenfalls mit der Integration in den Arbeitsmarkt; allerdings fokussiert er sich auf die polnischen Migranten und vergleicht diese Gruppe mit der größten Ausländergruppe in Deutschland - den Türken. Dabei bespricht Hönekopp sehr detailliert die Stellung polnischer Migranten auf dem Arbeitsmarkt in Deutschland Erwerbstätigkeit, Arbeitslosigkeit, Beschäftigung in Arbeitnehmer-Programm und Firmengründungen. Der weitere Kontext, in dem die Zuwanderung aus Polen besprochen wird, ist die Erwartung bezüglich des Volumens der Migration nach der EU-Osterweiterung, die auf Grund der makroökonomischen Migrationstheorien erörtert wurde. Ferner präsentiert Hönekopp die Übergangsregelungen bezüglich der Arbeitskraftzuwanderung in Deutschland im Vergleich zu anderen europäischen Länder, sowohl in der ersten als in der zweiten Phase, die die Integrationsmöglichkeiten beeinflussen.

Mit den Erwartungen bezüglich des Volumens der Migration aus Polen nach Westeuropa beschäftigt sich der Beitrag von Pawet Kaczmarczyk genauer. Er stellt die bedeutsamsten Prognosen dar und vergleicht sie mit den tatsächlichen Entwicklungen in der Auswanderung aus Polen in die ,alten“EUMitgliedstaaten nach der EU-Osterweiterung. Hier berücksichtigt er die Perspektive des Herkunftslands, die selten in den Studien präsent war. Dabei kann er zeigen, warum die meisten Prognosen der westeuropäischen Wissenschaftler falsch gelegen haben. Interessanterweise bespricht Kaczmarczyk auch die Defizite der verfügbaren statistischen Daten. Nichtsdestotrotz gelingt es ihm, einen Vergleich zwischen der polnischen Migration nach Deutschland und nach Großbritannien zu ziehen, um die dringende Frage zu beantworten, ob Deutschland den Wettbewerb um die „besten“ Migranten verliert.

Der letzte Beitrag in diesem Teil führt in die theoretische Grundlage für die weiteren Texte des Bands ein. Ludger Pries fragt, welche Auswirkungen die neuen Formen der Migration und ihre Dynamik auf die soziale Inkorporation von Migranten in der Ankunfts- und Herkunftsgesellschaft haben. Zugleich stellt er die unterschiedlichen Typen von Migration und die verschiedenen typischen Umgangsweisen mit den Ausländern in Europa dar und beschäftigt sich schließlich mit der Frage, ob eine gemeinsame Zuwanderungs- und Zugehörigkeitspolitik in der Europäischen Union entstehen kann. Dabei zeigt 
er am Beispiel Deutschlands, welche neuen Herausforderungen die transnationale Migration für die Nationalstaaten darstellt.

\section{Transnationale Verbindungen}

Der zweite Teil des Bands widmet sich den Verbindungen zwischen Polen und Deutschland, die durch die zirkuläre Migration, den Wunsch der Integration in die Aufnahmegesellschaft und des Beibehaltens der sozialen Bindungen zu der Herkunftsgesellschaft entstehen. Alle Beiträge nehmen eine transnationale Perspektive an. Die Ansätze zum Transnationalismus betrachten Migration nicht als einen ein- oder zweimaligen Wechsel zwischen zwei Orten sonder als einen Bestandteil kontinuierlicher Lebensläufe in Sozialräumen, die sich über Grenzen der Nationalstaaten erstrecken. Diese Perspektive ist auf Alltagsphänomene und soziale, politische und wirtschaftliche Beziehungen zwischen mehreren Orten gerichtet (Glick Schiller et al. 1992; Smith 1995, 1997; Goldring 1996, 1997, 2001; Basch et al. 1997; Smith/Guarnizo 1999; Faist 2000a; Pries 2001; Faist/Özveren 2004).

Die Humangeographin Birgit Glorius fokussiert sich in ihrem Beitrag auf das Phänomen transnationaler sozialer Räume am Beispiel polnischer Migranten in Leipzig. Sie untersuchte Aspekte der zirkulären Mobilität zwischen Polen und Deutschland, die kulturelle Alltagspraxis der Migranten, ihre Identitätskonstruktion und die gefühlte nationale Zugehörigkeit. Die Motive der Wanderung und Verbindungen zu Polen geben Anlass, über die Dauerhaftigkeit der Pendel- und die Chancen der Rückkehrmigration zu sprechen. Dabei zeigt Glorius die Variabilität transnationaler Lebensweisen und die innere Differenzierung transnationaler sozialer Räume auf. Ungewöhnlich und daher bemerkenswert ist der geographische Fokus auf eine ostdeutsche Stadt, in der die Zuwanderung andere Gründe und Traditionen hat und die sehr nah an Polen gelegen ist. Interessant ist auch die Methode der Untersuchung, in der sie qualitative Interviews (Fallstudien) mit einer quantitativen Befragung verbindet.

Andere Aspekte kommen in dem Beitrag von Agnes Senganata Münst zum Vorschein. Sie stellt die Ergebnisse einer Untersuchung der polnischen Migration in das Ruhrgebiet vor. Der Schwerpunkt dieser Studie richtete sich auf die Personen, die undokumentiert in privaten Haushalten arbeiten und zwischen Polen und Deutschland pendeln. Sie sind weder in das Gesundheitsnoch in das Steuersystem in Deutschland integriert. Der Beitrag bespricht zwei Typen von Frauen - eine mit langjähriger und eine mit kurzer Migrationsdauer. Dabei zeigt sich, dass alle Pendelmigrantinen in der Anfangsphase ihrer Migration von ethnischen Netzwerken profitieren, denn Personen polnischer Herkunft vermitteln ihnen die Kontakte zu Auftraggebern. Sie haben auch eine zentrale Bedeutung in dem Integrationsprozess in Deutschland. Der 
Ressourcentausch im ethnischen Netzwerk folgt sowohl den Regeln sozialer reziproker Beziehungen als auch einer Regel der kapitalistischen Dienstleistungsgesellschaft. Im Verlauf der Migration werden aber auch die sozialen Ressourcen der deutschen Auftraggeber wichtiger. Diese Netzwerke funktionieren ähnlich wie die ethnischen. Sie führen aber gleichzeitig zur Stabilisierung der Pendelmigration und der undokumentierten Arbeit in Deutschland.

Der Beitrag von Magdalena Nowicka und Maja Zielińska widmet sich einer bisher nie untersuchten Gruppe der polnischen Migranten, die die Niederlassungsfreiheit nutzten und sich für die Selbständigkeit entschieden haben. Es wird angenommen, dass eine berufliche Selbständigkeit nur mit einer gewissen Kenntnis des lokalen Marktes, ausreichendem Eigenkapital oder entsprechender Kreditwürdigkeit getroffen werden kann, was bereits ein bestimmtes Maß an Integration voraussetzt; sie deutet auch auf den Wunsch hin, in dem Land länger zu bleiben. Andererseits lässt sie eine Offenheit der Aufnahmegesellschaft vermuten. Die Autorinnen beschäftigen sich weniger mit den rechtlichen und ökonomischen Aspekten, sondern mit der transnationalen Verortung der Selbständigen und ihren Einfluss auf ihre soziale Lage in Deutschland und in Polen. Die Migration geht oft mit einem Statuswechsel einher, weil das Leben in unterschiedlichen Ländern mit unterschiedlichen sozialen Positionen verbunden ist. Eine transnationale Lebensführung gibt zusätzlich die Möglichkeit einer flexiblen Verortung und des Statuswechsels zwischen den Ländern und in einem der Länder. So wird Transnationalität zu einer wichtigen Ressource für die Verbesserung der eigenen sozialen Lage.

\section{Transnationale Inkorporationen}

Die weiteren zwei Beiträge verlassen die Mezo-Perspektive und widmen sich den Individuen und ihren Erzählungen. Hier werden zwei besondere Gruppen der Migranten besprochen - polnische Frauen, die in Deutschland in privaten Haushalten arbeiten und die Personen, die sowohl die polnische als auch die deutsche Staatsanghörigkeit besitzen. Beide Beiträge nutzen eine diskursanalytische Methode, um die Inkorporationsmuster näher zu untersuchen.

Dobrochna Katwa hat biographische Interviews mit in Polen lebenden Frauen durchgeführt, die zeitweise in Deutschland arbeiten. Sie stellt die Migration der Frauen im Kontext ihrer Stellung auf dem polnischen Arbeitsmarkt vor. Dabei bespricht sie kritisch auch die bisherige Migrationsforschung unter dem Aspekt des Genders. Die historische Perspektive dient ihr als Hintergrund für das Verstehen der Entwicklung der Geschlechtsrollen in der privaten Sphäre und ihre Veränderung auf Grund der 
Migration. Die durch die Migrantinnen angenommenen Geschlechtsrollen, die sie aus ihrem Herkunftsland kennen, beeinflussen auch die Beziehung, die sie zu ihren Arbeitsgebern in Deutschland entwickeln. Diese Rollen sind andererseits ein Mechanismus, mit dessen Hilfe die Migrantinnen die Schwierigkeiten des Alltags in Deutschland managen. Die Analyse erlaubt Kałwa die Frage zu stellen, inwieweit es überhaupt zu einem interkulturellen Austausch zwischen Aufnahme- und Herkunftsgesellschaften kommt.

Ewa Palenga-Möllenbeck beschäftigt sich mit den Identitäten und Zugehörigkeitsgefühlen der Angehörigen der vorwiegend in Oberschlesien ansässigen „deutschen Minderheit" in Polen, den so genannten „Doppelstaatler“, die sowohl in Polen als auch in Deutschland leben und in beiden Ländern Verwandte und Freunde haben. Auf Grund der deutschpolnischen Geschichte der Region Oberschlesien sind für diese Gruppe die Begriffe nationale oder ethnische Identität ambivalent. Die Geschichte nimmt eine besondere Stellung in den Erzählungen der Migranten ein, sowohl auf der Ebene der Region wie der eigenen Familie. In diesem Kontext wird auch die Erwerbsmigration als eine Unvermeidlichkeit verstanden. Dabei werden Schlesien und Deutschland gegenübergestellt und jeweils mit traditionellen gemeinschaftlichen Werten und der modernisierten westlichen Gesellschaft assoziiert. Die ethnischen Identitäten der von Palenga-Möllenbeck untersuchten Personen weisen eine große Vielfalt auf, wobei die regional gebundene Selbstbeschreibung als „Oberschlesier" eine Abgrenzung von den Deutschen als auch von den Polen dominiert. Auf dieser Basis beschreibt die Autorin die Migrationsprojekte der „Doppelstaatler“. Daraus wird deutlich, dass die Pendelmigration nicht hinreichend mit ökonomischen Ansätzen erklärt werden kann.

\section{Auswanderung und die Herkunftsregionen}

Die beiden letzten Beiträge beziehen sich ebenfalls auf die Besonderheiten der Migration aus Schlesien. Diese Region hat eine starke historische Bindung zu Deutschland. Vor allem aus der Region Oppeln kann man eine massive Auswanderung der autochthonen Population beobachten. Circa 25\% der Personen mit permanentem Wohnsitz in der Region Oppeln ist ausgewandert und lebt seit längerem im Ausland und weitere 34\% sind temporäre Migranten, die vor allem in Deutschland und Holland arbeiten. Heutzutage ist die Zahl der im Ausland arbeitenden Personen größer als die derjenigen, die in Polen beschäftigt sind. Dieser „Exodus“ hat erhebliche Folgen für die Herkunftsregion. Sie werden von den Autoren auf verschiedene Weise betrachtet.

Emilia Lewandowska und Tim Elrick vergleichen zwei Herkunftsgemeinden in Polen, eine in der Region Oberschlesien und eine in 
Ostpolen. Die Gemeinden charakterisieren eine andere Tradition der Migration und eine andere Art der Bindungen zum Ausland. Diese historischen sozialen Kontexte sind der Ausgangspunkt der Analyse der Auswirkungen der Mobilität ihrer Einwohner auf die Entwicklung der so genannten Migrationskulturen. Die Osterweiterung hatte Folgen für die Struktur, Dauer und Ziele der Auswanderung aus diesen Gemeinden. Die haben wiederum die sozialen Bindungen zwischen den Einwohnern der Gemeinden und ihre Einstellungen und Meinungen gegenüber den Migranten und der Migration allgemein beeinflusst. Sie mussten sich an die Situation der temporären Anwesenheit vieler Einwohner und Familienmitglieder anpassen. In jeder der Gemeinden entwickelte sich eine andere Migrationskultur. Ihre sozialen, ökonomischen, kulturellen und psychologischen Aspekte werden in dem Beitrag diskutiert.

Romuald Jończy präsentiert dagegen eine makroökonomische Perspektive für die Entwicklung der Region Oppeln. Die Arbeitsmigration hat in dieser Region das demographische und ökonomische Gleichgewicht ernsthaft zerstört. Der Beitrag zeigt, dass für die autochthone Bevölkerung in der Region Oppeln der primäre Arbeitsmarkt der deutsche und nicht der polnische ist. Die Erwerbswanderung nimmt ständig $\mathrm{zu}$ und die Arbeit im Ausland wird vordergründig von jungen Menschen aufgenommen. Die Wanderung wird allerdings nicht von Arbeitslosigkeit vorangetrieben, sondern ist ein Ergebnis der Fehlanpassung der Ausbildungs- und offenen Stellenstruktur in der Region. Ein weiteres Motiv der Arbeitsmigration ist ihre relative Rentabilität. Die regelmäßigen Geldtransfers der Migranten sowie ihre Abwesenheit in der Region wirken sich auf den Arbeitsmarkt und die Wirtschaftsentwicklung der Region negativ aus.

\section{Von Opfern zu Mitbürgern: Migration als Herausforderung für Europa}

Vor allem die Beiträge des letzten Teils des Buchs verdeutlichen, dass Migration und ihre Folgen im offenen europäischen Raum keineswegs nur ein Land betreffen. Auch aus deutscher Sicht ist die migrationsbedingte disharmonische Entwicklung bzw. Fehlentwicklung einer Region in Polen ein Problem. Deutschland ist und wird in den nächsten Jahren der größte Nettozahler der EU sein, Polen und seine Regionen sind zwar seit dem Beitritts Bulgariens und Rumäniens nicht mehr die ärmsten, das Land wird aber bis 2013 aus den EU-Struktur- und Kohäsionsfonds circa 59.5 Millionen Euro für die regionale Entwicklung erhalten (NSRO 2006). Die strukturelle Hilfe in den Jahren nach dem Beitritt (2004-2006) betrug 2.968 Millionen Euro, davon erhielt die kleine Woiwodschaft Oppeln 76.75 Millionen (Samorząd 2006). 
Auch der Beitrag von Paweł Kaczmarczyk zeigt, dass es Deutschland nicht egal sein kann, was auf den Arbeitsmärkten in Schweden, Irland oder Großbritannien passiert.

Was für den Ökonomen eine längst bekannte und recht gut erforschte Tatsache ist, wird von den Soziologen selten thematisiert und wenn, dann überwiegend als Zusammenstoß der Interessen und Kulturen und Desintegration in Europa (Bach 2000; Bös 2000; Immerfall 2000). Die Beiträge dieses Bands zeigen, dass es sich nicht um Konflikte, Spaltung oder Desintegration durch Migration handelt; allerdings erzwingt Migration neue Formen der Vergesellschaftung. Neben den nationalstaatlichen „sesshafteren“ Mehrheitsgesellschaften entwickeln sich in Europa mobile, transnationale Netzwerke, Identitäten und Lebensprojekte. Wie Birgit Glorius, Dobrochna Kałwa und Ewa Palenga-Möllenbeck zeigen, ist es möglich, das die Inkorporationsmuster und Identitäten sich weder an einer nationalen oder ethnischen Zugehörigkeit orientieren. Für die von den Autorinnen untersuchten Individuen sind die ethnischen oder nationalen Zugehörigkeiten weniger wichtig als ihre Arbeit, Freizeit und Freundschaften. Das Bewusstsein einer historischen Entwicklung und der Trennung der Nationen in Europa ist nur einer der vielen Aspekte, die ihre Identitäten und Praktiken mitkonstruieren. Für die Pendelmigranten ist es kein Widerspruch, sich mit beiden Nationen verbunden zu fühlen, aus beiden Ländern „das Beste“ zu schöpfen und gleichzeitig „fleißig wie ein Deutscher“ und „fantasievoll wie ein Pole“ zu sein, wie Palenga-Möllenbeck schreibt. Sie sind nicht sentimental über ihre nationale Zugehörigkeit, obwohl sie im engen Kontakt zu ihren Familien und Freunden im Herkunftsland stehen. In den Interaktionen in Deutschland beziehen sie sich dabei auf pragmatische Entscheidungen und zwischenmenschliche, universalistische Werte. Sie denken strategisch, wenn sie ihre Lebensräume über die nationalen Grenzen hinweg erstrecken. Sie sind auch pragmatisch, was die Integration in der Aufnahmegesellschaft angeht. Es kann für sie eigentlich vorteilhaft sein, weder in der Aufnahme- noch in der Herkunftsgesellschaft angepasst zu sein (Portes 1999: 229). Es scheint, dass viele Polen Mobilität und kulturelle Vielfalt als eine wertvolle Ressource anerkennen (Bennett 2001). Die Polen repräsentieren eine neue Art der existentiellen Kompetenz - sie nutzen die Verortung in beiden Ländern, um die eigenen Lebenschancen und die eigene soziale Positionierung selbst zu gestalten. Vielleicht sind die polnischen Migranten die „echten“ Europäer. Sie scheinen relativ einfach und flexibel auf die Veränderungen auf den Arbeitsmärkten zu reagieren; sie betrachten die Arbeit im Ausland als eine Antwort auf heutige transnationale Möglichkeiten und Gelegenheiten, eine Entscheidung, die zur jeder Zeit in der Zukunft erneut verhandelt werden kann. Diese Entscheidungen konstituieren die transnationale Dynamiken ihrer Lebensverläufe. 
Die alltäglichen Strategien der Arbeitsmigranten für ein besseres Leben und ihre transnationale Lebensräume haben bedeutsame Folgen für die nationalen Kulturen und Vorstellungen in Europa (Robins 2006: 26). Wie Stephen Castles (2003: 20) argumentierte, stellt die neue Logik der multiple Verortungen die zentrale Rolle des Nationalstaats für die sozialen Zugehörigkeiten in Frage. Die transmigratorischen Praktiken zwingen die Migranten zur ständigen Verhandlung der eigenen Zugehörigkeit und der eigenen Stellung in der Aufnahmegesellschaft (Aksoy/Robins 2000).

Die transnationalen Lebensräume der Migranten erschließen auch die immobilen Personen und Gruppen (Faist 2006: 53) und ihre Erweiterung erweckt bei den Mitgliedern der Aufnahmegesellschaften ein Gefühl des Verlusts der Kontrolle über das Geschehen. Die transmigratorischen Praktiken und zunehmend durchlässige Grenzen verlangen von den Aufnahmegesellschaften, ihr Verständnis der sozialen Integration neu zu verhandeln. Die Tatsache, dass sich durch die Migration die Erwartungs- und Vergleichshorizonte verändern (wie die Beiträge von Emilia Lewandowska und Tim Elrick, Ewa Palenga-Möllenbeck und Romuald Jończy zeigen) und dass die Verortung in mehreren Länder die individuelle soziale Positionierung (wie Magdalena Nowicka und Maja Zielińska analysierten) und die Erwartungen und Handlungen ganzer Gruppen (der Beitrag von Lewandowska und Elrick) verändern, beeinflusst das Empfinden von sozialer Gerechtigkeit und von Ungleichheiten. Dies wird wiederum darauf Einfluss haben, wie die Modalitäten der polnischen Präsenz in Deutschland verhandelt werden.

Migration ist der integrale Teil einer Vorstellung von der Welt - zum Beispiel als einen offenen Raum, in dem man sich ansiedeln kann - und deshalb wirkt sich darauf aus, wie sich Individuen, Gruppen, Nationen oder supranationale Formationen selbst und ihre Mit-Bürger vorstellen (Fortier 2006). Durch diese vielfältigen kognitiven Faktoren wird die Integration in Europa beeinflusst. Oft werden in der Europaforschung gerade diese kognitiven und diskursiven Elemente der sozialen Integration ignoriert, trotz des Postulats, Europa nicht nur als Integration von politischen, rechtlichen und wirtschaftlichen Systemen zu verstehen.

Für polnische Migranten innerhalb der EU ist die Erstreckung der eigenen Lebensräume über die nationalstaatlichen Grenzen zunehmend eine Selbstverständlichkeit. An diesem Prozess nehmen auch die Mitglieder der Aufnahmegesellschaften teil - zum Beispiel gehören auch die Deutschen zu den sozialen Netzwerken, die die Migration aufrecht halten, wie Senganata Münst beschreibt. Allerdings teilen sie mit den Migranten nicht gleichermaßen die Vorstellung eines gemeinsamen Lebensraums, wie die Zeitungsmeldungen und die Politikgestaltung zeigen.

Die Herausforderung der Migration liegt daran, dass Migranten die vorgegebenen Räume - die nationalstaatlichen Räume - überschreiten und ihre 
Lebensräume und sozialen Beziehungen über die Grenzen hinweg konstituieren. Diese Herausforderung betrifft sowohl die Aufnahme- und Herkunftsgesellschaften und die nationalstaatliche Politik als auch die Wissenschaft, die angemessene Instrumente zur Untersuchung dieser grenzüberschreitenden Prozesse ansetzen muss. Eine Einschätzung der prominenten Wissenschaftler ist, dass der Wissenschaft noch immer eine angemessene Terminologie und Methodologie zur Beschreibung dieser Phänomene fehlt (Beck 2001, 2004; Beck/Sznaider 2006; Robins 2006). Die Transnationalismus- und Migrationsforschung ist in dieser Hinsicht der Europaforschung voraus, weil sie die nationale Perspektive verlässt. Die Autoren der Beiträge in diesem Band haben ihre Analysen bei den sozialen Beziehungen und Identitäten angesetzt und erst im zweiten Schritt interpretiert, inwieweit sie von ,nationalem“ beeinflusst werden. Sie haben sie zuerst ohne den „nationalen Rahmen“ untersucht, diesen aber später einbezogen, um die Ergebnisse zu verstehen. So machten sie die Prozesse sichtbar, die sich über die nationalen Grenzen erstrecken.

Die gleiche „Denkübung“ kann man für Europa einsetzen: Sich Europa als einen gemeinsamen Lebensraum für mehrere Nationen und ethnische Gruppen vorzustellen bedeutetet, die sozialen Beziehungen auf einer anderen räumlichen Skala zu denken, ohne dabei die temporären Aspekte (kollektive Erinnerungen und Identitäten) zu vergessen. Diese Vorstellungsfähigkeiten, sowohl der Gesellschaften als auch der Wissenschaft, werden über die zukünftige Gestalt Europas entscheiden. 


\section{Literatur}

Aksoy, Asu /Robins, Kevin (2000): »Thinking across Spaces: Transnational Television from Turkey«. European Journal of Cultural Studies 3(3), S. 54363.

Aksoy, Asu/ Robins, Kevin (2003): »Banal transnationalism: the difference that television makes «. In: Karim H. Karim (Hg.), The media of diaspora, London, Routledge, S. 89-104.

Bach, Maurizio (2000): »Die Europäisierung der nationalen Gesellschaften? Problemstellungen und Perspektiven einer Soziologie der europäischen Integration«. In: ders. (Hg.), Die Europäisierung nationaler Gesellschaften. Sonderheft 40 der Kölner Zeitschrift für Soziologie und Sozialpsychologie, S. 11-35.

Bade, Klaus/Oltmer, Jochen (1999): Deutsche Einwanderer aus Osteuropa, Osnabrück: IMIS.

Basch, Linda/Glick Schiller, Nina/Szanton Blanc, Cristina (1997): Nations Unbound. Transnational Projects, Post-colonial Predicaments, and Deterritorialized Nation-States, Amsterdam: Gordon and Breach.

Bauer, Ingrid/Ehmer, Josef/Hahn, Sylvia (Hg) (2002): Walz-MigrationBesatzung. Historische Szenarien des Eigenen und Fremden, Klagenfurt/Celovec, Bundesministerium für Bildung, Wissenschaft und Kultur.

Beck, Ulrich (2000): »The Cosmopolitan Perspective: Sociology of the Second Age of Modernity« The British Journal of Sociology 51(1), S. 79-105.

Beck, Ulrich (2004): Der kosmopolitische Blick.Oder: Krieg ist Frieden, Frankfurt a.M.: Suhrkamp.

Beck, Ulrich/Grande, Edgar (2004): Das kosmopolitische Europa, Frankfurt am Main: Suhrkamp.

Beck, Ulrich/Sznaider, Natan (2006): »Unpacking cosmopolitanism for the social sciences: a research agenda«. British Journal of Sociology 57(1), S. 1-23.

Bennett, Tony (2001): Differing diversities: cultural policy and cultural diversity, Strasbourg, Council of Europe Publishing.

Berliner Zeitung (04.05.2005): Invasion der Fliesenleger. Wie polnische Handwerker ganz legal den Berliner Arbeitsmarkt durcheinander wirbeln, von Marcel Gäding.

Bös, Matthias (1993): »Ethnisierung des Rechts? Staatsbürgerschaft in Deutschland, Frankreich, Großbritannien und den USA«. Kölner Zeitschrift für Soziologie und Sozialpsychologie 45(4), S. 619-643. 
Bös, Mathias (2000): »Zur Kongruenz sozialer Grenzen. Das Spannungsfeld von Territorien, Bevölkerungen und Kulturen in Europa«. In: Maurizio Bach (Hg.), Die Europäisierung nationaler Gesellschaften. Sonderheft 40 der Kölner Zeitschrift für Soziologie und Sozialpsychologie, S. 429-455.

Breuer, Michael/Faist, Thomas/Jordan, Bill (1994): Club Theory, Migration and Welfare States, ZeS-Arbeitspapier 15/94, Bremen: Centre for Social Policy Research, Universität Bremen.

Bielefeld, Ulrich (Hg) (1998): Das Eigene und das Fremde. Neuer Rassismus in der Alten Welt, Hamburg: HIS.

Castles, Stephen (2003): »Towards a sociology of forced migration and social transformation«. Sociology 37(1), S. 13-34.

Cyrus, Norbert (1994): »Polnische Arbeitsmigranten in der Bundesrepublik Deutschland. Skizze eines Systems ausdifferenzierter Pendelmigration. «In: Zbigniew Kurcz/Władysław Misiak (Hg.), Mniejszość niemiecka w Polsce i Polacy w Niemczech, Wrocław: Wydawnictwo Uniwersystetu Wrocławskiego, S. 179-193.

Die Zeit (12/2005): Die Billig-Unternehmen. Handwerker aus Osteuropa drängen auf den deutschen Markt oft als Scheinselbständige, von Ulrike Meyer-Timpe. Eder, Klaus (2000): »Konstitutionsbedingungen einer transnationalen Gesellschaft in Europa. Zur nachholenden Modernisierung Europas“. In: Wolfgang Heyde/Thomas Schaber (Hg.), Demokratisches Regieren in Europa, BadenBaden: Nomos, S. 87-102.

European Foundation for the Improvement of Living and Working Conditions (1990): Mobility and Social Cohesion in the European Community: A Forward Look, Dublin: European Foundation.

Faist, Thomas (2000): The Volume and Dynamics of International Migration and Transnational Social Spaces, Oxford: Oxford University Press.

Faist, Thomas/ Özveren, Eyüp (Hg.) (2004): Transnational Social Spaces: Agents, Networks and Institutions, Aldershot: Avebury.

Faist, Thomas (2006): »Cultural logics of transnational migration«. In: Kevin Robins (Hg.), The challenge of transcultural diversities. Transversal study on the theme of cultural policy and cultural diversity, Strasbourg: Council of Europe, S. 51-68.

Fortier, Anne-Marie (2006): »The Politics of Scaling, Timing and Embodying: Rethinking the 'New Europe'«. Mobilities 1(3), S. 313-333.

Frankfurter Allgemeine Zeitung (17.05.2005): Lohndumping. Für Hungerlohn filetieren Polen das „Russenfleisch“, von Siegfried Thielbeer. 
Gazeta Wyborcza (26.04.2006): Wrocław ściaga polskich pracowników z....Anglii, von Michał Kokot.

Gazeta Wyborza (27.06.2006): Ciemna liczba szukających pracy w UE, von Patrycja Maciejewicz.

Gazeta Wyborcza (27.06.2006): Kontrole biją w polskie firmy w Niemczech, von Marek Wielgo.

Gazeta Wyborcza (28.07.2006): PO chce ulg, aby zatrzymać zarobkowa emigrację, von Leszek Kostrzewski und Piotr Skwirowski.

Gazeta Wyborcza (26.10.2006): Młodzi Polacy nie muszą być tanią siłą roboczą UE (PAP-Meldung).

Gazeta Wyborcza (31.01.2007): Germanizacja dzieci? Skargi Polaków w Brukseli, von Konrad Niklewicz.

Glick Schiller, Nina/Basch, Linda/ Szanton Blanc, Cristina (Hg.) (1992): Towards a Transnational Perspective on Migration: Race, Class, Ethnicity, and Nationalism Reconsidered, New York: New York Academy of Sciences.

Glick Schiller, Nina/Basch, Linda/ Szanton Blanc, Cristina (1995): »From immigrant to transmigrant: theorizing transnational migration«. Anthropological Quarterly 68(1), S. 48-63.

Goldring, Luin (1996): »Blurring the Border: Transnational Community and Social Transformation in Mexico-U.S. Migration«. In: Dan A. Chekki (Hg.), Research in Community Sociology CI, Stanford/London: JAI Press, S. 69-104. Goddring, Luin (1997): »Power and Status in Transnational Social Spaces«. In: Ludger Pries (Hg.), Transnationale Migration (Sonderband 12 der Zeitschrift Soziale Welt), Baden-Baden: Nomos, S. 179-196.

Goldring, Luin (2001): »Disaggregating Transnational Social Spaces: Gender, Place and Citizenship in Mexico-U.S. Transnational Spaces«. In: Ludger Pries (Hg.), New Transnational Social Spaces. International Migration and Transnational Companies, London: Routledge, S. 59-76.

Hahn, Alois (1994): »Die soziale Konstruktion des Fremden«. In: Walter Sprondel (Hg.) Die Objektivität der Ordnung und ihre kommunikative Konstruktion, Frankfurt am Main: Suhrkamp, S. 140-163.

Heckmann, Friedrich/Tomei, Verónica (Hg.) (1996): Freizügigkeit in Europa. Migrations- und europapolitische Aspekte des Schengen-Vertrages, Bonn: Europa Union Verlag.

Immerfall, Stefan (2000): »Fragestellungen einer Soziologie der europäischen Integration«. In: Maurizio Bach (Hg.), Die Europäisierung nationaler Gesellschaften. Sonderheft 40 der Kölner Zeitschrift für Soziologie und Sozialpsychologie, S. 481-503. 
Jordan, Bill/Düvell, Franck (2002): Irregular Migration. The Dilemmas of Transnational Mobility, Cheltenham/Northampton, MA: Edward Elgar.

Krasnodębski, Zdzisław (2001): »Die polnische Minderheit in Deutschland als Forschungsobjekt«. In: Zdzisław Krasnodębski/Nele Krampen (Hg.), Polen in Bremen. Eine unsichtbare Miderheit? Bremen: Universität Bremen-KUA, S. 13-55.

Lehmann, Rainer/Peek, Rainer/Gänsfuß, Rüdiger/Husfeld, Vera (2000) LAU 9. Aspekte der Lernausgangslage und der Lernentwicklung. Klassenstufe 9. Ergebnisse einer längschnittlichen Untersuchung in Hamburg, Hamburg: Hamburger Bildungsserver.

Lie, John (1995): »From International Migration to Transnational Diaspora«. Contemporary Sociology 24(4), S. 303-306.

Miera, Frauke (1997): »Migration aus Polen. Zwischen nationaler Migrationspolitik und transnationalen sozialen Lebensräumen«. In: Hartmut Häußermann/Ingrid Oswald (Hg.), Zuwanderung und Stadtentwicklung (Leviatan Sonderheft 17), Opladen: Westdeutscher Verlag, S. 232-254.

Morokvašic, Mirjana/Tunguy, Anne, de (1993): »Between East and West: A New Migratory Space«. In: Anne de Tunguy/Mirjana Morokvašic (Hg.), Bridging states and markets: international migration in the early 1990s, Berlin: edition sigma, S. 245-263.

Mrowka, Heinrich (2000): ",Polonia“-Organisationen und ihre deutschen Ansprechpartner«. In: Anna Wolff-Powęska/Eberhard Schluz (Hg.), Polen in Deutschland. Integration oder Separation?, Düsseldorf: Droste Verlag, S. 213236.

NSRO (2006): Polska. Narodowe Strategiczne Ramy Odniesienia 2007-2013 wspierające wzrost gospodarczy i zatrudnienie. Narodowa Strategia Spójności, Warszawa: Ministerstwo Rozwoju Regionalnego.

Portes, Alejandro (Hg.) (1995): The economic sociology of immigration: essays on networks, ethnicity, and entrepreneurship, New York: Russell Sage Foundation.

Pries, Ludger (Hg.) (2001): New Transnational Social Spaces. International Migration and Transnational Companies, London: Routledge.

Radke, Frank-Olaf (1996): »Fremde und Allzufremde. Prozesse der Ethnisierung gesellschaftlicher Konflikte«. Links 28, S. 20-22.

Samorząd (2006): Wykorzystanie wybranych krajowych i europejskich programów pomocowych wdrażanych w województwie opolskim, Opole: Samorząd Województwa Opolskiego. 
Sheffer, Gabriel (1996): » A New Field of Study: Modern Diasporas in International Politics«. In: Gabriel Sheffer (Hg.) Modern Diasporas in International Politics, London: St. Martin's Press, S. 1-15.

Silbereisen, Rainer/Landermann, Ernst-Dieter/Schmitt-Rodermund, Eva (Hg.) (1999): Aussiedler in Deutschland. Akkulturation von Persönlichkeit und Verhalten, Opladen: Leske + Budrich.

Smith, Michael Peter/Guernizo, Luid Eduardo (Hg.) (1999): Transnationalism from Below, New Brunswick, NJ/London: Transaction Publishers.

Stern (13/2005): Die Lohn-Schlachter, von Marion Losse.

Stern (17/2005): Extra: Ein Jahr danach. Handwerker aus Polen - 1000 ganz legale Alleskönner, von Bert Heinzelmeier.

Straubhaar, Thomas (2000): Why do we need a General Agreement on Movements of People (GAMP)? Discussion Paper 94, Hamburg: HWWA.

Strobl, Rainer/Kühnel, Wolfgang (2000): Dazugehörig und ausgegrenzt. Analysen zu Integrationschancen junger Aussiedler, Weinheim/München: Juventa Verlag.

Verordnung (EWG) Nr. 1408/71 des Rates vom 14. Juni 1971 zur Anwendung der Systeme der sozialen Sicherheit auf Arbeitnehmer und Selbständige sowie deren Familienangehörige, die innerhalb der Gemeinschaft zu- und abwandern.

Verwiebe, Roland (2004): Transnationale Mobilität innerhalb Europas. Eine Studie zu den sozialstrukturellen Effekten der Europäisierung, Berlin: edition sigma. 